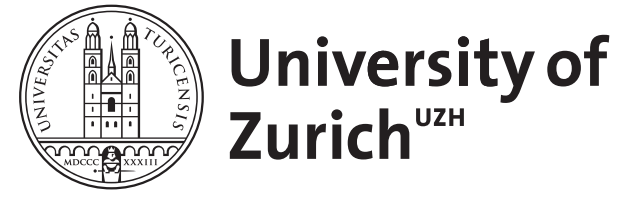
Archive

University of Zurich

University Library

Strickhofstrasse 39

CH-8057 Zurich

www.zora.uzh.ch

Year: 2012

\title{
Leihmutterschaft als persönliche Beziehung
}

Bleisch, Barbara

DOI: https://doi.org/10.1515/jfwe.2012.17.1.5

Posted at the Zurich Open Repository and Archive, University of Zurich

ZORA URL: https://doi.org/10.5167/uzh-188104

Journal Article

Published Version

Originally published at:

Bleisch, Barbara (2012). Leihmutterschaft als persönliche Beziehung. Jahrbuch für Wissenschaft und Ethik, 17(1):5-28.

DOI: https://doi.org/10.1515/jfwe.2012.17.1.5 


\title{
Leihmutterschaft als persönliche Beziehung
}

\author{
von Barbara Bleisch
}

\begin{abstract}
Ist Leihmutterschaft moralisch zu rechtfertigen, oder geht dieses Verfahren notwendig mit einer Ausbeutung oder einer Instrumentalisierung der Leihmutter einher? In diesem Beitrag wird argumentiert, dass eine Leihmutter nur dann in moralisch unzulässiger Weise instrumentalisiert wird, wenn sie auf ihre Funktion, ein Kind für ein anderes Paar auszutragen, reduziert wird. Um einer solchen Reduktion vorzubeugen, sollten Leihmutterschaftsverhältnisse als persönliche triadische Beziehungen zwischen Bestelleltern, austragender Mutter und Kind verstanden werden. Eine solche anspruchsvolle Konzeptualisierung von Leihmutterschaft nimmt auch das Kindeswohl in den Blick, das in der Debatte bisher zu wenig berücksichtigt wurde.
\end{abstract}

Keywords: Leihmutterschaft, Instrumentalisierung, Menschenwürde, triadische Beziehung, Kindeswohl, geteilte Elternschaft.
Kinderkriegen im globalen Zeitalter ist keine Frage der Natur mehr - sondern der Logistik. Florian Hanig ${ }^{1}$

Der familienrechtliche Grundsatz „Pater semper incertus est, mater semper certa est" ist spätestens seit der Debatte um die Leihmutterschaft ins Wanken geraten. ${ }^{2}$ Während andere Verfahren der modernen Reproduktionsmedizin wie die In-vitroFertilisation (IVF), die Präimplantationsdiagnostik (PID) oder die Samenspende nicht an diesem Grundsatz zu rütteln vermochten, tut dies die Praxis der Leihmutterschaft in klarer Weise. Als Mutter eines Kindes wird gewöhnlich jene Frau

\section{HANIG 2011, 147.}

2 Ob bereits die Eizellspende den Rechtsgrundsatz „Mater semper certa est“ erschüttert, ist umstritten: Vor der Zeit der modernen Reproduktionsmedizin wurde der Grundsatz deskriptiv verstanden: Eine Frau konnte gar kein anderes Kind gebären, als das (genetisch) ihre. Insofern falsifizierte das Aufkommen der Eizellspende den deskriptiven Grundsatz, denn eine Frau kann heute ein Kind gebären, das genetisch nicht mit ihr verwandt ist. Der Grundsatz kann aber auch normativ verstanden werden: Als rechtliche Mutter eines Kindes soll immer jene gelten, die das Kind geboren hat. Diesem normativ verstandenen Grundsatz widerspricht die Eizellspende nicht. Die Leihmutterschaft widerspricht ihm jedoch insofern, als die gebärende Leihmutter (zumindest der Idee nach) nicht die rechtliche Mutter des Kindes sein wird. 
anerkannt, die das Kind ausgetragen und geboren hat. Im Fall der Leihmutterschaft ist die Sache weniger eindeutig: Eine Frau gebärt ein Kind, dessen Mutter sie nicht ist, wenngleich sie mit dem Kind schwanger war und es zur Welt brachte. Dieser Umstand hat etwas Irritierendes an sich. Zwar gibt es seit jeher Kinder, die sowohl über eine biologische, als auch über eine soziale Mutter verfügen, die nicht identisch sind: So ist die Adoptiv- oder Stiefmutter nicht dieselbe wie die leibliche Mutter eines Kindes, die ihre Rechte als Mutter nach der Geburt an eine andere Frau abgetreten hat. Irritierend ist in Bezug auf die Leihmutterschaft also nicht der Umstand, dass sich zuweilen eine andere Person um ein Kind kümmert und als erziehungsberechtigt auftritt als jene, die es geboren hat. Bemerkenswert ist vielmehr, dass die Frau, die das Kind austrägt, offenbar nicht mit dem eigenen, sondern mit dem Baby eines anderen Paares schwanger ist. Die gebärende Frau tritt damit lediglich als wörtlich zu nehmende - „Gebärmutter“ auf, in der das Wunschkind eines anderen Paares heranwächst.

Dieser Beitrag geht der genannten Irritation auf den Grund und fragt nach den moralischen Implikationen der Leihmutterschaft: Ist Leihmutterschaft moralisch zu rechtfertigen? Oder geht dieses Verfahren notwendig mit einer Ausbeutung oder Instrumentalisierung der Leihmutter einher? Wie ist Leihmutterschaft überhaupt zu verstehen: als eine Erwerbsarbeit oder als Form der Mutterschaft, die neben anderen Arten der Mutterschaft Bestand haben sollte? Welchen Blick auf die Leihmutter und auf das Kind, das entsteht, legen welche Verständnisse von Leihmutterschaft nahe - und welches Verständnis ist aus moralischer Sicht zu verteidigen und zu fördern?

Nach einigen terminologischen Klärungen (1.) eröffne ich meine Überlegungen mit einer Diskussion von zwei Einwänden gegen die Leihmutterschaft, die in der Debatte besonders im Hinblick auf kommerzielle Leihmutterschaften öfters vorgebracht werden: Einerseits wird behauptet, diese Praxis stelle eine Ausbeutung der Leihmutter dar (2.); andrerseits würden Leihmütter unzulässig instrumentalisiert (3.). Ich werde argumentieren, dass beide Einwände zwar für viele Formen der gegenwärtig real existierenden Leihmutterschaftsverhältnisse zutreffen, dass sie aber nicht als genereller Einwand gegen jede Form von Leihmutterschaft Bestand haben. Eine Leihmutter wird nämlich nur dann instrumentalisiert, wenn sie auf ihre Funktion, ein Kind für ein anderes Paar auszutragen, reduziert wird. In einem nächsten Schritt (4.) mache ich einen positiven Vorschlag für ein Verständnis von Leihmutterschaft, demzufolge Leihmütter gerade nicht auf ihre Funktion des Austragens und Gebärens von Kindern reduziert werden. Mein Vorschlag lautet, dass Leihmutterschaftsverhältnisse aus Gründen der Moral als persönliche triadische Beqiehungen und Leihmutterschaft als eine Form der Mutterschaft verstanden werden sollten. Die Leihmutterschaft als persönliche Beziehung zwischen Bestelleltern, austragender Mutter und Kind geht einher mit einer Idee der geteilten Elternschaft, in der Leihmutter und Bestelleltern je ihre eigene Rolle ausfüllen. Eine solche Konzeptualisierung von Leihmutterschaft rückt auch das Wohl des Kindes, das aus der Leihmutterschaft hervorgeht, in den Blick und begegnet damit einer Lücke in der Debatte: Das Kind als Ziel einer jeden Leihmutterschaft wurde bisher nämlich noch kaum berücksich- 
tigt. Dieser Beitrag diagnostiziert damit ein doppeltes Defizit in der bioethischen Diskussion um Leihmutterschaften: Die Leihmutterschaft wurde bislang zu sehr als Dienstleistungs- oder Arbeitsverhältnis, und nicht als persönliche Beziehung verstanden; und das Wohl des Kindes, um dessen willen die Leihmutterschaften überhaupt zustande kommen, spielte eine zu marginale Rolle.

Am erfolgreichen Zustandekommen einer Leihmutterschaft ist stets auch mindestens ein Arzt oder eine Ärztin, welche die IVF sowie den Embryotransfer vornehmen, beteiligt. Die ethische Perspektive der behandelnden Ärzte lasse ich in diesem Beitrag jedoch undiskutiert. ${ }^{3}$ Ebenfalls undiskutiert lasse ich die Frage, ob es homosexuellen Paaren erlaubt sein soll, Leihmütter zu engagieren. Da ich keinen Grund sehe, weshalb homosexuelle Paare hinsichtlich Fragen der Reproduktion und Elternschaft anders behandelt werden sollten als heterosexuelle Paare, werde ich auf dieses Thema an dieser Stelle nicht weiter eingehen. ${ }^{4}$

\section{Terminologische Klärungen}

Grundsätzlich lassen sich vier Facetten von Mutterschaft unterscheiden, die im traditionellen Familienbild auf einer Frau vereint sind: i) Die genetische Mutterschaft bezeichnet das genetische Abstammungsverhältnis: Die genetische Mutter eines Kindes wird mit dem Sperma des genetischen Vaters befruchtet oder tritt als Eizellspenderin auf; ii) die austragende Mutterschaft (engl. „gestational motherhood“) umfasst Schwangerschaft und Geburt; iii) die soziale Mutterschaft betrifft das Zusammenleben mit dem Kind und seine Erziehung; iv) die rechtliche Mutterschaft ist identisch mit dem Sorgerecht für das Kind. Der Begriff der „biologischen Mutter“ wird im Folgenden vermieden, weil er in der Debatte sowohl für die genetische als auch für die austragende Mutter verwendet wird. Eine Leihmutter ist also per definitionem stets die austragende, nie jedoch die soziale Mutter des Kindes, welches sie geboren hat. Je nach Variante der Leihmutterschaft ist die Leihmutter überdies die genetische Mutter des betreffenden Kindes. Allein dieses Auseinanderklaffen der verschiedenen Aspekte der Mutterschaft mag irritieren. Es wird jedoch unsere Realität und un-

Für eine Diskussion der Rolle des Arztes siehe z. B. BER 2000, 156 ff.

4 Zugunsten einer Ungleichbehandlung von homosexuellen Paaren gegenüber heterosexuellen Paaren wird meist ins Feld geführt, das entsprechende Kind würde unter seiner Familiensituation leiden, weil die gegenwärtige Gesellschaft homosexuelle Paare als Eltern noch nicht hinreichend anerkenne. Gegen dieses Argument kann eingewendet werden, dass a) ein empirischer Beleg dieser Behauptung aussteht; dass b) durch die diskriminierende Praxis die Akzeptanz dieser alternativen Familienmodelle nicht gefördert, sondern weiter unterdrückt wird; und dass c) andere ungewöhnliche Familienkonzepte wie bspw. solche, in denen ein Elternteil stark behindert ist, ebenfalls mit Verweis auf den Schutz des Kindeswohls verboten werden müssten. 
sere Bilder von Familie immer stärker prägen, insofern als die Patchworkfamilie, in der zumindest soziale und genetische Elternteile nicht mehr identisch sind, neben dem herkömmlichen Familienmodell der Kernfamilie an Bedeutung stetig zulegt. ${ }^{5}$

Es lassen sich zwei Typen von Leihmutterschaft unterscheiden: In der traditionellen Leibmutterschaft wird die Leihmutter künstlich mit dem Sperma des angehenden sozialen Vaters befruchtet. Die austragende Mutter ist also zugleich die genetische Mutter des Kindes. Sie muss deshalb bei der Geburt auf ihre Fürsorgerechte, die ihr als genetische Mutter zustehen, verzichten (analog zur Freigabe eines Kindes zur Adoption), und die soziale Mutter muss das Kind adoptieren. Die traditionelle Leihmutterschaft wird vermutlich schon seit Langem praktiziert, denn sie ist nicht auf die modernen Mittel der Reproduktionsmedizin angewiesen. Die heute meistdiskutierte Form der Leihmutterschaft ist die austragende Leihmutterschaft: Die Leihmutter trägt dabei einen Fötus aus, der genetisch nicht der ihre ist. Ihr wird also ein IVF-gezeugter Embryo eingepflanzt. Dabei sind vier Untervarianten der austragenden Leihmutterschaft möglich in Abhängigkeit davon, ob die Keimzellen komplett, teilweise oder gar nicht von den zukünftigen sozialen Eltern stammen (siehe Tabelle).

\section{Tabelle: Varianten der Leihmutterschaft}

\begin{tabular}{|l|l|l|l|l|l|}
\hline & Leihmutter & $\begin{array}{l}\text { Genetische } \\
\text { Mutter }\end{array}$ & $\begin{array}{l}\text { Genetischer } \\
\text { Vater }\end{array}$ & Soziale Mutter & Sozialer Vater \\
\hline $\begin{array}{l}\text { Traditionelle } \\
\text { Leihmutterschaft }\end{array}$ & $\begin{array}{l}\text { Identisch mit } \\
\text { genetischer } \\
\text { Mutter }\end{array}$ & $\begin{array}{l}\text { Identisch } \\
\text { mit Leih- } \\
\text { mutter }\end{array}$ & $\begin{array}{l}\text { Späterer } \\
\text { sozialer } \\
\text { Vater }\end{array}$ & $\begin{array}{l}\text { Keine genetische } \\
\text { Verwandtschaft } \\
\text { mit dem Kind }\end{array}$ & $\begin{array}{l}\text { Identisch mit } \\
\text { genetischem } \\
\text { Vater }\end{array}$ \\
\hline $\begin{array}{l}\text { Austragende } \\
\text { Leihmutterschaft, } \\
\text { Typ 1 }\end{array}$ & $\begin{array}{l}\text { Keine gene- } \\
\text { tische Ver- } \\
\text { wandtschaft } \\
\text { mit dem Kind }\end{array}$ & $\begin{array}{l}\text { Spätere } \\
\text { soziale } \\
\text { Mutter }\end{array}$ & $\begin{array}{l}\text { Späterer } \\
\text { sozialer } \\
\text { Vater }\end{array}$ & $\begin{array}{l}\text { Eizelle stammt } \\
\text { von ihr; Embryo } \\
\text { IVF-gezeugt }\end{array}$ & $\begin{array}{l}\text { Samenzelle } \\
\text { stammt von ihm; } \\
\text { Embryo IVF- } \\
\text { gezeugt }\end{array}$ \\
\hline $\begin{array}{l}\text { Austragende } \\
\text { Leihmutterschaft, } \\
\text { Typ 2 }\end{array}$ & $\begin{array}{l}\text { Keine geneti- } \\
\text { sche Ver- } \\
\text { wandtschaft } \\
\text { mit dem Kind }\end{array}$ & $\begin{array}{l}\text { Fremde } \\
\text { Eizell- } \\
\text { spenderin }\end{array}$ & $\begin{array}{l}\text { Späterer } \\
\text { sozialer } \\
\text { Vater }\end{array}$ & $\begin{array}{l}\text { Keine genetische } \\
\text { Verwandtschaft } \\
\text { mit dem Kind }\end{array}$ & $\begin{array}{l}\text { Samenzelle } \\
\text { stammt von ihm; } \\
\text { Embryo IVF- } \\
\text { gezeugt }\end{array}$ \\
\hline $\begin{array}{l}\text { Austragende } \\
\text { Leihmutterschaft, } \\
\text { Typ 3 }\end{array}$ & $\begin{array}{l}\text { Keine geneti- } \\
\text { sche Ver- } \\
\text { wandtschaft } \\
\text { mit dem Kind }\end{array}$ & $\begin{array}{l}\text { Spätere } \\
\text { soziale } \\
\text { Mutter }\end{array}$ & $\begin{array}{l}\text { Fremder } \\
\text { Samen- } \\
\text { spender }\end{array}$ & $\begin{array}{l}\text { Eizelle stammt } \\
\text { von ihr; Embryo } \\
\text { IVF-gezeugt }\end{array}$ & $\begin{array}{l}\text { Keine genetische } \\
\text { Verwandtschaft } \\
\text { mit dem Kind }\end{array}$ \\
\hline $\begin{array}{l}\text { Austragende } \\
\text { Leihmutterschaft, } \\
\text { Typ 4 }\end{array}$ & $\begin{array}{l}\text { Keine geneti- } \\
\text { sche Ver- } \\
\text { wandtschaft } \\
\text { mit dem Kind }\end{array}$ & $\begin{array}{l}\text { Fremde } \\
\text { Eizell- } \\
\text { spenderin }\end{array}$ & $\begin{array}{l}\text { Fremder } \\
\text { Samen- } \\
\text { spender }\end{array}$ & $\begin{array}{l}\text { Keine genetische } \\
\text { Verwandtschaft } \\
\text { mit dem Kind }\end{array}$ & $\begin{array}{l}\text { Keine genetische } \\
\text { Verwandtschaft } \\
\text { mit dem Kind }\end{array}$ \\
\hline
\end{tabular}

Bei der austragenden Leihmutterschaft von Typ 2 und 4 sind Eizellspenden vonnöten. In einigen Ländern ist zwar die Samenspende, nicht jedoch die Eizellspende erlaubt. ${ }^{6}$ Die Frage, ob und inwiefern bereits die Eizellspende an sich moralisch prob-

5 Vgl. CAPREZ 2012.

${ }^{6}$ So z. B. in Deutschland, Italien, Österreich und in der Schweiz. Zum rechtlichen Verbot der Eizellspende in Deutschland siehe KELLER et al. 1992. 
lematisch ist, lasse ich in diesem Beitrag undiskutiert. Die rechtliche Zulässigkeit der Leihmutterschaft ist weltweit sehr unterschiedlich geregelt und die Rechtslage ändert sich laufend: Einige Länder wie bspw. Dänemark, Italien, Schweden, Deutschland, Österreich und die Schweiz verbieten dieses Verfahren; andere Ländern binden die legale Leihmutterschaft an gesetzliche Vorgaben (bspw. die Ukraine, Großbritannien, Israel oder Georgien) oder lassen sie ohne staatliche Regulierung zu. ${ }^{7}$

\section{Zur Gefahr der Ausbeutung}

Die meisten Leihmütter, die gegenwärtig ihre Dienste anbieten, tun dies in Erwartung finanzieller Vorteile. Viele von ihnen stammen aus Schwellenländern, etwa aus der Ukraine, aus Südafrika oder aus Indien. ${ }^{8}$ Der Umstand, dass sich offenbar vor allem Frauen aus Schwellen- und Entwicklungsländern als austragende Mutter zur Verfügung stellen, nährt den Eindruck, bei der Leihmutterschaft handle es sich um eine moralisch verwerfliche Form der Ausbeutung und Instrumentalisierung von Frauen. ${ }^{9}$ Dieser Verdacht verstärkt sich durch die Verwendung pejorativer Bezeichnungen von Leihmüttern als „Mietobjekt Gebärmutter“ oder „Reproduktionstanks".10 Die Tatsache, dass relativ arme Frauen sich vermögenderen Paaren als Leihmütter anbieten, ist jedoch, wie ich gleich ausführen werde, für sich genommen sicherlich kein hinreichendes Kriterium für Ausbeutung durch diejenigen, die entsprechende Angebote nachsuchen. Zudem müssen sich Frauen ganz offensichtlich nicht zwingend allein aus finanziellen Motiven als Leihmütter anbieten: So stellen sich Frauen in altruistischen Leihmutterschaftsverhältnissen bspw. aus Gründen der Freundschaft oder der Anteilnahme am Schicksal kinderloser Paare für das Austragen eines fremden Kindes zur Verfügung; auf diese Möglichkeit komme ich später zurück.

Bei der kommerziellen Leihmutterschaft stehen primär materielle Motive im Vordergrund. Aus dem Umstand, dass eine Person etwas vor allem aus finanziellen Gründen tut, folgt allerdings nicht bereits, dass diejenigen, die ihre Tätigkeit in Anspruch nehmen, sie ausbeuten. ${ }^{11}$ Ein solches Kriterium würde implizieren, dass sich

Für eine Diskussion der rechtlichen Lage siehe HöRNLE (im Druck).

8 Vgl. dazu bspw. das ukrainische Internetportal „La vita felice“ für Leihmutterschaften URL http://mother-surrogate.com/ (Stand: 08.05.2012) oder das südafrikanische Fertilitätszentrum „Medfem“ URL http://www.medfem.co.za (Stand: 08.05.2012).

9 Vgl. zu diesem Verdacht z. B. FIELD 1989, 25; ERLINGER 2011, 156; ANDERSON 1990 und für eine Diskussion HörNLE (im Druck), Abschnitt IV.

10 Vgl. demgegenüber den Versuch einer sprachlichen Neutralisierung der Praxis in der Rede von ,Ersatzmutterschaft ${ }^{\star}$ anstelle von ,Leihmutterschaft', etwa in HöRNLE (im Druck) und WIESEMANN 2006.

11 So auch SCHABER 2010, $144 \mathrm{f}$. 
ein Paar durchaus von einer Leihmutter helfen lassen darf, wenn diese altruistisch motiviert ist, nicht jedoch, wenn diese als Gegenleistung Geld verlangt und ohne entsprechende Entschädigung nicht einwilligen würde, das Kind auszutragen. Das kann nicht überzeugen. Schließlich müsste bei Annahme dieses simplen Kriteriums nahezu jede Erwerbsarbeit als moralisch fragwürdig eingestuft werden, denn viele würden ihrer Arbeit nicht länger nachgehen, wenn sie dafür nicht bezahlt würden. Eine Ausbeutung muss also zusätzliche Kriterien erfüllen. Welche diese sind, ist umstritten und hängt vom zugrunde gelegten Konzept von Ausbeutung ab. Tatsächlich wird der Begriff der Ausbeutung in politischen Debatten oft als rhetorisches Totschlagargument eingesetzt, ohne dass klar würde, worin die Ausbeutung genau besteht und auf welches Konzept von Ausbeutung sich das Urteil bezieht. ${ }^{12}$ Jemanden auszubeuten heißt, ganz allgemein gesprochen, in unfairer Weise von dieser Person zu profitieren, wobei die Unfairness am Ergebnis oder am Prozess festgemacht werden kann. In erster Linie kann man deshalb unterscheiden zwischen Konzepten von Ausbeutung, die das Tauschergebnis, und solchen, die den Tauschprozess als ausbeuterisch disqualifizieren. Eine Möglichkeit, das Tauschergebnis als Ausbeutung zu bezeichnen, besteht darin, die Ausbeutung als einen Tausch von Gütern zu verstehen, der die ausgebeutete Person schädigt. Eine Ausbeutung wird dann als eine Situation vorgestellt, in der die eine Partei sich etwas nimmt, was der anderen Partei zustünde. ${ }^{13}$ Begünstigt wäre also nur eine Partei, nämlich jene, die ausbeutet, während die ausgebeutete Person leer ausgeht bzw. der ihr zustehenden Güter beraubt wird. Ein solcher Begriff von Ausbeutung greift allerdings zu kurz, denn eine Ausbeutung kann durchaus für beide involvierten Parteien Vorteile haben und - im Vergleich zur Situation vor der Ausbeutung - beide Parteien besser stellen. In vielen Szenarien, die als paradigmatische Ausbeutungsfälle gelten, ist die Ausbeutung sogar gerade deshalb möglich, weil die ausgebeutete Person letztlich mehr Vorteile aus dem Tauschhandel zieht als die ausbeutende Person. So erklären sich bspw. billige Arbeitskräfte in Schwellenländern bereit, für einen sehr geringen Lohn zu arbeiten, weil für sie - im Gegensatz zu den Arbeitgebern - mehr, nämlich ihre Existenzsicherung auf dem Spiel steht. Gerade der Umstand, dass eine Partei ein weitaus gröBeres Interesse am Zustandekommen der Transaktion hat, erleichtert offenbar in vielen Fällen eine Ausbeutung. ${ }^{14}$ Dies bestätigt sich auch mit Blick auf Leihmutter-

$12 \mathrm{Zu}$ verschiedenen Konzepten von Ausbeutung siehe z.B. WERTHEIMER 2008.

13 Vgl. TORMEY 1974, 207.

14 Vgl. zu dieser These Wertheimer 2008. Dieser Problematik wird bspw. in Forschungsprojekten am Menschen insofern Rechnung getragen, als nicht beliebig hohe Entschädigungen für ein Forschungsvorhaben bezahlt werden dürfen, um die Attraktivität am Versuch teilzunehmen und damit die Gefahr einer Ausbeutung einzudämmen; siehe dazu bspw. die Rechtslage in der Schweiz, Art. 14 HFG; vgl. zu diesem Punkt auch die Stellungnahme 397 des American Congress of Obstetricians and Gynecologists (ACOG) zu Leihmutterschaft (URL http://www.acog.org/Search?Keyword=Surrogacy (Stand: 14.5.2012)). 
schaftsverhältnisse: Während viele Frauen aus finanziellen Gründen hoffen, als Leihmutter engagiert zu werden, steht das Auftrag gebende Paar, das aus einem Überangebot von Leihmüttern wählen kann, weniger unter Zugzwang, was eine Ausbeutung durch Dumpingpreise erst recht möglich macht.

Daran wird zweierlei ersichtlich: Erstens lässt sich allein aus dem Umstand, dass ein Arbeitsverhältnis (wie auch eine Leihmutterschaft) zum Vorteil beider Parteien ist, nicht schon schließen, dass es sich dabei nicht um einen Fall von Ausbeutung handelt. Zweitens scheint das große Interesse der potentiell ausgebeuteten Person am Zustandekommen eines Vertragsabschlusses nur dann problematisch, wenn ihr Interesse missbraucht wird, etwa indem eine zu geringe Entschädigung entrichtet wird. Tatsächlich machen einige ein Ausbeutungsverhältnis denn auch an der Unfairness des Preises fest, der für das fragliche Gut oder für die Leistung entrichtet wird. Dieser Ansatz ist allerdings mit dem Problem konfrontiert, dass ganz generell unklar ist, nach welchen Kriterien gerechte Preise festgesetzt werden sollen. Die diesbezüglichen Schwierigkeiten, die sich mit Blick auf handelsübliche Güter wie Baumwolle oder Kaffee ergeben, stellen sich erst recht, wenn handelsunübliche Güter wie bspw. Organe, Eizellen oder Leihmutterschaften kommodifiziert werden sollen. ${ }^{15}$ Nun könnte man gegen das Herausstreichen dieser Schwierigkeit einwenden, dass der Markt den Preis bei einer Legalisierung der Leihmutterschaft regeln wird, denn schließlich gilt für jedes gehandelte Gut, dass kein ,objektiver Preis‘ dafür existiert, der sich am gehandelten Gut festmachen ließe, sondern dass dieser Preis in einem fairen Prozess ausgehandelt werden muss. Damit wird das zweite Konzept von Ausbeutung in den Blick genommen, das auf den Tauschprozess fokussiert.

Mit Blick auf eine moralische Bewertung der Leihmutterschaft als Tauschprozess erweist sich m.E. Allan Buchanans Verständnis von Ausbeutung als zielführend, der das Ausbeutungsverbot mit dem Instrumentalisierungsverbot verknüpft. Buchanan versteht unter einer Ausbeutung eine Handlung, die Personen unzulässig instrumentalisiert. „To exploit a person involves the harmful, merely instrumental utilization of him or his capacities, for one's own advantage or for the sake of one's own ends. " 16 Leihmutterschaften gelten in philosophischen Debatten neben dem Organhandel oder der Folter denn auch oft als paradigmatische Fälle von unzulässigen Instrumentalisierungen von Personen. In der Diskussion des Instrumentalisierungsverbots wird dabei oft auf Immanuel Kants Selbstzweckformel verwiesen, die verlangt, dass man andere wie auch sich selbst nie bloß als Mittel, sondern immer auch als Zweck

15 Im ukrainischen Leihmutterschaftszentrum „La vita felice“ wird das Austragen eines Kindes je nachdem, ob eigene Keimzellen eingebracht werden, mit ca. 6.000 US\$ entlohnt, hinzu kommen Gelder für Versicherung, Nahrung, Umstandskleidung, etc.; siehe URL http://mother-surrogate.com/price.html (Stand: 10.5.12).

16 BUCHANAN 1985, 87. 
behandeln soll. ${ }^{17}$ Dabei besteht ein innerer Zusammenhang zur (Miss)Achtung der Menschenwürde: Wer das Instrumentalisierungsverbot verletzt, verstößt, so die Idee, gegen die unantastbare Würde des Menschen. ${ }^{18}$ Wir können also gemäß einer Zweiteilung der Zweckformel ${ }^{19}$ erstens fragen, ob die Praxis der Leihmutterschaft Frauen notwendig zum bloßen Mittel degradiere; und wir können zweitens fragen, ob die Bestelleltern die Leihmutter unmöglich auch als Zweck an sich behandeln können.

\section{Zu den Gefahren von Instrumentalisierung und Menschenwürdeverletzung}

Beginnen wir mit der ersten Frage, ob Personen, die ein Kind von einer Leihmutter austragen lassen, die Leihmutter in unzulässiger Weise benutzen oder instrumentalisieren. In Leihmutterschaften wie auch beim Verkauf lebend gespendeter Organe benutzen Personen einander offenkundig als Mittel zu einem Zweck: Ein Paar oder eine Einzelperson nutzen die Dienste einer Leihmutter, um sich den Kinderwunsch zu erfüllen. Allerdings benutzt auch die Leihmutter das Auftrag gebende Paar: Sie kann sich dank deren Kinderwunsch finanzielle Vorteile verschaffen. Dieses gegenseitige Nutzenverhältnis oder Instrumentalisieren hat offenbar so lange nichts moralisch Anstößiges, als sich beide Parteien freiwillig dafür entscheiden, sich auf den Handel einzulassen. Samuel Kerstein schlägt im Zusammenhang mit dem Verkauf eigener lebend gespendeter Organe vor, die Idee der Freiwilligkeit so zu verstehen, dass eine Person der Transaktion genau dann freiwillig zustimme, wenn es für sie als autonome Person auch möglich wäre, ihr nicht zuzustimmen. ${ }^{20}$ Solange sich also beide Parteien auch gegen das Arrangement entscheiden können, behandeln sie einander, so Kerstein, nicht als bloßes Mittel und instrumentalisieren einander nicht in moralisch unzulässiger Weise. Wann kann sich aber eine Person unmöglich autonom gegen ein Angebot entscheiden? Sicherlich ist dies dann der Fall, wenn eine Entscheidung entweder unter Täuschung oder unter Zwang erfolgt. Im Folgenden

17 KANT 1785, AA 429. Die Frage, ob sich eine Frau dadurch, dass sie als Leihmutter arbeitet, selbst instrumentalisiert und damit eine moralische Pflicht gegen sich selbst verletzt, diskutiere ich in diesem Beitrag nicht.

18 Zum Zusammenhang zwischen Instrumentalisierung und Verletzung der Menschenwürde, siehe z. B. SCHABER 2010.

${ }^{19} \mathrm{Ob}$ eine solche Zweiteilung die korrekte Interpretation der kantischen Selbstzweckformel ist, ist umstritten. Für eine solche Zweiteilung spricht sich bspw. SCHABER 2010 aus.

${ }^{20}$ Kerstein spricht in diesem Zusammenhang vom „possible consent account“ (KERSTEIN 2009, 152 ff.). 
untersuche ich, was Täuschung und Zwang im Hinblick auf die Arbeit als Leihmutter bedeuten könnten.

Was im Fall der Einwilligung in Leihmutterschaft als Täuschung gilt, ist umstritten. Klarerweise müssen Leihmütter mit den Risiken einer Schwangerschaft bekannt gemacht werden, und es bedarf transparenter Abmachungen, was die Bestelleltern von der Leihmutter an Vorsorge für den Fötus erwarten (bspw. hinsichtlich Ernährung, Lebensweise, medizinischen Untersuchungen, etc.). Einige Autoren argumentieren darüber hinaus, die Täuschung bestünde bereits darin, dass v.a. Erstgebärende gar nicht wissen könnten, worauf sie sich als Leihmutter einlassen, denn das Gefühl einer Schwangerschaft zu antizipieren, sei unmöglich. ${ }^{21}$ Insofern involviere jede Leihmutterschaft insbesondere bei Erstgebärenden eine Täuschung. Diese Unmöglichkeit, die Schwangerschaft zu antizipieren, betrifft weniger die physischen Veränderungen, die mit einer Schwangerschaft einhergehen, als den Umstand, dass ein individueller Mensch in einem heranwächst, der sich im Laufe der Schwangerschaft immer stärker bemerkbar macht und zu dem sich - selbst wenn sich die Betroffene dagegen wehrt - auch Gefühle entwickeln können (vgl. Abschnitt 4). Als Beleg für diese These wird ins Feld geführt, dass es selbst zweitgebärenden Leihmüttern schwer fallen könne, sich nach der Geburt von ihrem Baby zu trennen, weil sie sich während der Schwangerschaft stärker als vermutet mit dem Kind zu identifizieren begannen. ${ }^{22}$ Doch einerseits könnte einem dies auch mit anderen Projekten zustoBen, die einem am Herzen liegen: Denken wir bspw. an einen Künstler, der sich von einem Auftragswerk nach Vollendung nicht trennen mag, weil ihm das Kunstwerk im Laufe der Zeit so wichtig geworden ist. Das Problem, dass wir uns also in einer Arbeit auf ein Projekt so sehr einlassen, dass wir uns danach nur noch schwerlich von ihm trennen können, scheint nicht spezifisch für das Verhältnis einer Leihmutter zum bestellten Kind. Allerdings besteht ein gewichtiger Unterschied zwischen der Situation des Künstlers und derjenigen der Leihmutter: Der Künstler kann sich immer noch entscheiden, sein Kunstwerk zu behalten (und die finanzielle Busse hinzunehmen). Eine Leihmutter dagegen hat in vielen Fällen diese Option nicht: Einerseits hat sie zuweilen keine rechtliche Handhabe, das Sorgerecht für das ausgetragene Kind zu erhalten. Diese rechtliche Situation ließe sich mit entsprechenden Gesetzen allerdings anpassen, etwa indem eine Regelung geschaffen würde ähnlich wie im Fall der Freigabe von Kindern zur Adoption in Deutschland: Das entsprechende Gesetz verlangt, dass ein Kind erst acht Wochen nach der Geburt zur Adoption freigegeben werden kann. Bis zu diesem Zeitpunkt kann sich eine

21 Vgl. z. B. BER 2000, 159.

22 In diesem Zusammenhang berühmt geworden ist der US-amerikanische Fall „Baby M“, bei dem nach einer traditionellen Leihmutterschaft ein Sorgerechtsstreit um das Kind entbrannte, der in letzter Instanz zugunsten des Vaters entschieden wurde; vgl. dazu z. B. TIEU 2009. 
Frau also weiterhin entscheiden, ihr Kind $\mathrm{zu}$ behalten. ${ }^{23}$ Ich komme auf diesen Punkt in Abschnitt 4 zurück. Andererseits dürften sich faktisch die wenigsten Leihmütter selbst dann, wenn sie rechtlich das Sorgerecht für das Neugeborene erhalten könnten, in der Lage befinden, von diesem Recht auch tatsächlich Gebrauch machen zu können. Ein Kind aufzuziehen bedeutet einen entscheidenden Einschnitt in der Lebensgestaltung, zu dem sich eine Leihmutter aus praktischen Gründen möglicherweise gar nicht entscheiden kann: Vielleicht will ihr Partner kein (weiteres) Kind oder sie würde ihre Stelle verlieren, wenn sie nicht wie ausgemacht binnen einer kurzen Frist an den Arbeitsplatz zurückkehren würde, oder sie ist finanziell nicht in der Lage, das Kind großzuziehen. Insofern ist eine Leihmutter nicht gleichermaßen frei wie ein Künstler, sein Werk zu behalten, an dem ihm mehr liegt, als er vor Abschluss des Auftrags dachte.

Wie steht es mit dem zweiten von Kersteins Kriterien für eine freiwillige Entscheidung, nämlich mit der Abwesenheit von Zwang? Betrachten wir folgenden Vorschlag: Eine Frau kann sich für oder gegen die Erwerbsarbeit, welche die Leihmutterschaft für sie darstellt, frei entscheiden, wenn sie entweder finanziell so gut gestellt ist, dass sie auf das Geld nicht vital angewiesen ist (weil ihre eigenen oder die Grundbedürfnisse ihrer Kinder bereits gedeckt sind), oder aber wenn sie einer anderen Erwerbsarbeit nachgehen könnte, die ihre eigenen und die Grundbedürfnisse ihrer Kinder ebenfalls zu decken vermöchte, wenn also eine annehmbare Alternative zur Verfügung steht. ${ }^{24}$ Sind diese Bedingungen nicht erfüllt, ist eine Frau insofern nicht frei darin, sich gegen ihre Arbeit als Leihmutter zu entscheiden, als sie aus materieller Not heraus agiert und in der Leihmutterschaft das einzige Mittel sieht, sich aus der Armut zu befreien. Tatsächlich dürfte dies hinsichtlich vieler real vorkommender kommerzieller Leihmutterschaften der Fall sein: Einkommensschwache Frauen aus Entwicklungs- oder Schwellenländern bieten sich als Leihmütter an in der Hoffnung, mit dem Einkommen bspw. ihren Kindern eine Schulausbildung zu ermöglichen oder sich aus der Armut zu befreien. Nun ist allerdings fraglich, ob diese Art der ,Unfreiwilligkeit' bereits etwas moralisch Problematisches an sich hat. Denn effektiv gilt für viele Erwerbsmöglichkeiten Ähnliches: Denken wir etwa an eine Lehrerin, die auf ihr Gehalt vital angewiesen ist und über keine annehmbare berufliche Alternative verfügt. Sie ist also gezwungen, als Lehrerin zu arbeiten; sie kann sich gemäß obigem Kriterium nicht frei entscheiden, ihre Arbeit einfach aufzugeben, und ihre Anstellung käme gemäß Kersteins Kriterium somit einer Instrumentalisierung ihrer Person gleich. Würden wir ihre Arbeitsverhältnisse als moralisch problematisch ansehen? Wohl kaum. Wollen wir also an der These festhalten, dass es moralisch von besonderer Brisanz ist, wenn eine Frau sich aus rein materiel-

${ }^{23}$ Vgl. $\int 1747$ Abs. 3 BGB sowie Wiesemann 2006, 148, die die Wichtigkeit dieses Erfordernisses ebenfalls betont.

${ }^{24}$ Was es heißt, auf etwas vital angewiesen zu sein und was zu den Grundbedürfnissen zählt, die eine alternative Beschäftigung decken müsste, bedürfte einer genauen Definition. 
len Gründen als Leihmutter anbietet, müssen wir einen zusätzlichen Grund dafür angeben, weshalb die Arbeit als Leihmutter im Vergleich zu anderen Erwerbstätigkeiten besonders problematisch sein könnte. Denn die Arbeit einer Leihmutter scheint sich von der Arbeit einer Lehrerin doch in relevanter Hinsicht zu unterscheiden. Es muss also moralische Bedenken gegen die Leihmutterschaft geben, die nichts mit der eben skizzierten Art von Unfreiwilligkeit zu tun haben. Welcher Art diese sein könnten, wird klarer, wenn wir den zweiten Teil der Kant'schen Zweckformel genauer betrachten.

Der zweite Teil der Zweckformel verlangt, andere stets auch als Zweck an sich zu behandeln. Damit meint Kant, dass dem Gegenüber ein unbedingter und unvergleichbarer Wert zugestanden wird; für ihn gleichbedeutend mit der Idee, dass die Würde des Gegenübers geachtet wird. Die Würde eines Menschen hat somit keinen Preis: Sie kann nicht gegen andere Güter abgewogen oder zugunsten eines anderen Werts geopfert werden. So wäre nach Kant beispielsweise die Tötung einer Person zur Rettung von zwei anderen Personen nicht zu rechtfertigen, denn der Wert zweier Personen kann gegen den Wert einer einzelnen Person nie abgewogen werden. ${ }^{25}$ Als paradigmatischer Fall einer Menschenwürdeverletzung wird oft die Sklaverei genannt: Ein Mensch hat als Sklave einen Preis, er wird verdinglicht und zur austauschbaren Ware. Ein Sklave wird nicht als ein Zweck an sich behandelt; seine Würde ist verletzt.

Verdinglicht die Praxis der Leihmutterschaft Frauen und stellt sie damit eine Verletzung der Menschenwürde dar? Tatsächlich kommt ein solcher Verdacht nur mit Blick auf bestimmte Erwerbsarbeiten oder Tätigkeiten auf. Bei der oben erwähnten Arbeit als Lehrerin stellt sich das Problem einer möglichen Verdinglichung nicht oder nicht in gleicher Weise (auch wenn sie möglicherweise aufgrund eines zu niedrigen Lohnes ausgebeutet werden kann). Sobald jedoch die körperliche Integrität einer Arbeitskraft tangiert ist, stellt sich die Frage offenbar unmittelbar: Die Person ist als ganze weitaus stärker mit ihrer Arbeit oder ihrer Tätigkeit ,vermischt", als wenn sie lediglich ihre Fertigkeiten, ihre Ideen oder auch die Erledigung einer Aufgabe gegen Bezahlung zur Verfügung stellt. Deshalb wird der Verdacht der Verdinglichung oder Würdeverletzung bei Arbeiten, in die eine Person in körperlich intimer Weise involviert ist wie bei Organspenden, Prostitution oder Leihmutterschaft, besonders häufig geäußert. ${ }^{26}$ Auch zur Beantwortung der Frage nach einer Verdinglichung der Leihmutter erweist sich der Blick auf den ähnlich gelagerten Fall der Lebendorganspende als hilfreich. Samuel Kerstein vertritt in diesem Zusammenhang die These, dass die Frage nach einer Würdeverletzung sich nicht prinzipiell, sondern nur unter Bezugnahme auf den Kontext, innerhalb dessen sich der Organverkauf abspielt, beantworten lasse. Verkaufe eine Person ihre Organe allein aus Gründen der Armut und sei sie auf den vereinbarten Erlös angewiesen, so nähre

25 Siehe KANT 1785, AA, 429 und KANT 1797, AA VI, 462. Vgl. auch SCHABER 2012, $40 \mathrm{f}$.

26 Vgl. Pateman 1983 und Teman 2010, 1-3. 
dies den Verdacht, sie stünde als ganze Person zum Verkauf, so Kerstein. Sie kann nicht mehr frei über ihren Körper verfügen. Bei einer altruistischen Lebendorganspende dagegen, die durchaus auch materiell abgegolten werden kann in Form einer Entschädigung oder einer Geste des Dankes, die aber nicht aufgrund von finanzieller Not erfolgt, verhält sich die Sache Kerstein zufolge anders: In einem solchen Fall könne man nicht behaupten, jemand erhalte einen Preis und werde als Mittel zum Zweck benutzt. ${ }^{27}$ Denn die Organspenderin könnte sich in letzterem Szenario jederzeit auch anders entscheiden. Weil dies dem potentiellen Organempfänger bewusst ist, ist sein Blick auf die Organspenderin jener auf einen gleichwertigen Partner, während im ersten Fall der Blick einer abhängigen Person gilt, die nicht frei über ihren eigenen Körper verfügen kann, sondern diesen ,einzusetzen' gezwungen ist. Gegen Kerstein lässt sich an dieser Stelle allerdings einwenden, dass die Organknappheit und die damit einhergehende Abhängigkeit der Organempfänger von ihren potentiellen Organspendern die Machtverhältnisse genau umdrehe und insofern gerade die umgekehrte Gefahr einer Instrumentalisierung des Patienten durch den Organverkäufer drohe. Eine solche Umkehrung der Verhältnisse trifft jedoch gegenwärtig sicher nicht auf Leihmutterschaften zu, weil sich aktuell mehr Leihmütter kommerziell anbieten als nachgefragt werden. Übertragen auf die Praxis der Leihmutterschaft, scheint an Kersteins These denn auch effektiv etwas dran zu sein: Es macht für die moralische Beurteilung der hier diskutierten Praxis einen Unterschied, ob die Leihmutter finanziell relativ gut gestellt ist oder ob die Leihmutter aus einer finanziellen Not heraus agiert. Die finanzielle Not ist dabei - und dies ist der springende Punkt - nicht nur deshalb moralisch relevant, weil bei einer notleidenden Leihmutter zumindest das Risiko besteht, dass sie ausgebeutet oder instrumentalisiert wird (1. Teil der Zweckformel), sondern auch, weil die Gefahr größer ist, dass ihre Würde verletzt wird, weil der Blick des Auftrag gebenden Paars auf sie der Blick auf eine käufliche Person sein kann (2. Teil der Zweckformel). Ob die Auftrag gebende Partei die Leihmutter tatsächlich als käuflich ansieht, als eine Person also, die für einen Preis zu haben ist, ist - folgt man Kerstein - letztlich eine empirische Frage, die von Gesellschaft zu Gesellschaft differiert. ${ }^{28}$ Die moralische Erlaubtheit von Leihmutterschaften hängt also - nimmt man Kersteins Idee ernst - davon ab, wie die Leihmutter in der Gesellschaft respektive vom Bestellpaar wahrgenommen wird: als eine Person, die als ganze für einen Preis zu haben ist - oder als eine Person, die eine bestimmte Fähigkeit einbringt, für die sie bezahlt wird. Eine Fähigkeit einzubringen, heißt weitaus mehr, als seinen Körper zur Verfügung zu stellen. Entsprechend sollte auch die Schwangerschaft einer Leihmutter aufgrund des Instrumentalisierungsverbotes als eine Tätigkeit verstanden werden, die weitaus mehr umfasst, als das Dienen als Reproduktionstank.

Kerstein liefert also vor dem Hintergrund der Kant'schen Zweckformel ein negatives Kriterium für die kommerzialisierte Lebendorganspende: Diese ist moralisch

27 Vgl. KeRSTEIN 2009, 161.

28 Bei KeRstein am Beispiel des Organverkaufs erläutert (KERSTEIN 2009, 162). 
nicht zulässig, wenn sie die Zweckformel insofern verletzt, als der Organverkauf eine Handlung darstellt, in der eine Person als ganze zum Verkauf steht. Ich habe dieses Kriterium auf Leihmutterschaften übertragen und argumentiert, dass das Engagieren einer Leihmutter dann illegitim ist, wenn mit dieser Handlung zum Ausdruck gebracht wird, dass die Leihmutter auf ihre Funktion als Gebärmutter reduziert wird und entsprechend als Person einen Preis erhält. Im Folgenden möchte ich mit Blick auf den zweiten Teil der Zweckformel einen positiven Vorschlag dafür machen, wie Leihmutterschaftsverhältnisse konzipiert und ausgestaltet werden könnten, sodass die Leihmütter in ihnen als Zweck an sich, als Personen geachtet werden können.

\section{Leihmutterschaft als persönliche triadische Beziehung}

Mein Vorschlag lautet, dass Leihmutterschaftsverhältnisse aus Gründen der Moral als persönliche Beziehungen im Unterschied zu Dienstleistungsverhältnissen verstanden und dementsprechend ausgestaltet werden sollten. ${ }^{29}$ Unter einer persönlichen Beziehung verstehe ich eine nahe Beziehung zwischen Menschen, die sich nicht nur als Vertragspartner oder aufgrund einer bestimmten Rolle, die sie füreinander einzunehmen in der Lage sind, sondern auch als Personen schätzen und achten und denen an dieser Verbindung liegt. Eine Leihmutter wird also in einer persönlichen Beziehung zwischen ihr und den intendierten Eltern nicht allein aufgrund des Umstands geschätzt, dass sie in der Lage ist, das Wunschkind auszutragen, sondern auch als Person. ${ }^{30}$ Diese persönliche Beziehung ist dabei als triadische Beziehung vorzustellen: eine Relation zwischen dem Auftrag gebenden Paar, der Leihmutter sowie dem Kind, das die Leihmutter austrägt. ${ }^{31}$ Der hier gemachte Vorschlag schlägt damit gegenüber vielen bisherigen Konzepten von Leihmutterschaften eine doppelte Korrektur vor: Einerseits ist ein Leihmutterschaftsverhältnis aus Gründen der Moral nicht als ein Vertrags- oder Arbeitsverhältnis zu verstehen, das nach Ablauf des Vertrags - in diesem Fall: nach der Geburt - endet, sondern als eine persönliche Beziehung, die andauert, damit alle Beteiligten ihre Verantwortung, die dieser Beziehung entspringt, übernehmen können. Andererseits trägt das triadische Konzept der Tatsache Rechnung, dass an einer Leihmutterschaft nicht nur zwei, sondern drei Parteien in einer moralisch bedeutsamen Weise beteiligt sind. ${ }^{32}$ Effektiv wurde das

29 WiESEMANN 2006 deutet in ihrer Arbeit einen ähnlichen Weg an.

30 Axel Honneth und Beate Rössler sprechen von persönlichen Beziehungen deshalb auch von „Person-qua-Person-Beziehungen“ (HONNETH, RÖSSLER 2008, 10 ff).

31 Das Konzept der Triade hat auch van den AKKER 2007 fruchtbar gemacht.

32 Wie in der Einleitung erwähnt, sind am Zustandekommen einer Leihmutterschaft weitere Personen wie bspw. Ärzte beteiligt. Sie sind jedoch nicht in moralisch bedeutsamer 
Kind, das den eigentlichen Zweck einer Leihmutterschaft darstellt, in der betreffenden bioethischen Diskussion um Leihmutterschaften bislang weitgehend außer Acht gelassen. ${ }^{33}$ Der Fokus der Debatte lag bisher vielmehr auf den Interessen und Rechten von Leihmüttern und intendierten Eltern. Schwangerschaft und Geburt betreffen jedoch nicht allein Erwachsene, die Kinder gebären oder sich solche wünschen, sondern immer auch mindestens ein Kind - und zwar in existentieller Weise, denn es existiert gerade und ausschließlich aufgrund dieser Leihmutterschaft.

Warum Leihmutterschaften nicht als Arbeitsverhältnisse, sondern als persönliche Beziehungen konzipiert werden sollten, wird deutlich, wenn wir die einzelnen Komponenten des Leihmutterschaftsverhältnisses in den Blick nehmen. Beginnen wir mit der Relation zwischen dem Kind und der Leihmutter: Während der Schwangerschaft ist diese Verbindung zumindest körperlich von einer Intimität, wie sie nicht zu überbieten ist. ${ }^{34}$ Es ist schlichtweg keine andere Arbeit denkbar, in die eine Person körperlich so stark involviert ist, wie wenn ihr Blutkreislauf mit demjenigen einer anderen Person verbunden ist. Außerdem hat diese Verbindung extreme körperliche Auswirkungen: In keiner anderen Phase des Lebens verändert sich eine Person so schnell und so offensichtlich hinsichtlich Gewicht, Essgewohnheiten, Schlafverhalten bis hin zu Fortbewegungsarten wie während der neun Monate einer Schwangerschaft. ${ }^{35}$ Selbst wenn die Leihmutter den Umstand ihrer körperlichen Veränderung als Auswirkung eines Jobs deutet, wird und sollte sie dies nicht darüber hinwegtäuschen, dass ein Mensch in ihr heranwächst, der zumindest physiologisch ganz und gar von ihr abhängt. Aus dieser Abhängigkeit allein ergibt sich allerdings noch keine Pflicht. Denn wie Judith Jarvis Thomson mit ihrem Geiger-Beispie ${ }^{6}$ überzeugend zeigt, sind wir nicht verantwortlich für von uns abhängige Wesen allein aufgrund dieser Abhängigkeit. Entsprechend könnte man mit konventionellen Modellen der Leihmutterschaft als Dienstleitungsverhältnis argumentieren, die Leihmutter schulde nicht dem Fötus einen sorgsamen Umgang mit ihm, sondern sie schulde diese Sorge den Bestelleltern, die sie für eine erfolgreiche Schwangerschaft be-

Weise involviert und nicht Teil der hier angemahnten persönlichen Beziehung, in deren Zentrum die Leihmutterschaft steht.

33 Zu diesem Schluss kommt auch TONG 2003, 369. Eine Ausnahme bilden hier bspw. die Richtlinien des Wissenschaftlichen Beirats der Bundesärztekammer zur Durchführung der assistierten Reproduktion von 1998, in denen das Kindswohl auch im Hinblick auf die so genannte geteilte Mutterschaft bezweifelt wird.

34 Vgl. dazu GHEAus 2011, Abschnitt 5 und ausführlich Muldin 2005 sowie WiesEMANN 2006.

35 Vgl. Mullin 2005, 67.

36 Thomson verteidigt mit diesem Gedankenexperiment die Abtreibung, indem sie eine ungewollt Schwangere mit einer Frau vergleicht, die ohne ihre Einwilligung an den Blutkreislauf eines berühmten Geigers angeschlossen worden ist, weil nur sie seine Blutgruppe teilt und er ohne Unterstützung eines fremden Blutkreislaufes sterben würde (THOMSON 1971). 
zahlen, die in der Geburt eines gesunden Kindes resultiert. In Studien gaben Leihmütter denn auch an, auf ihre Gesundheit zu achten und das Kind schädigende Einflüsse zu vermeiden, weil sie fürchteten, dass sie - wenn das Kind nicht gesund zur Welt käme - die vereinbarte Bezahlung nicht erhalten würden. ${ }^{37}$ Die Vorstellung, dass die Leihmutter Fürsorge und Achtsamkeit nicht dem Kind schulde, sondern den Bestelleltern, ist jedoch eine Verdrehung der Pflichtverhältnisse: In erster Linie ist die Sorge dem werdenden Kind als heranwachsendem Menschen geschuldet, und nicht den späteren sozialen Eltern, die sich dieses Kind möglichst gesund wünschen. Dasselbe trifft auch für eine Nanny zu, die auf ein Kind achtgibt: In erster Linie ist sie dem Kind verpflichtet, für seine Sicherheit und sein Wohlbefinden zu sorgen; die entsprechende Obhutspflicht ist ihr von ihren Arbeitgebern übertragen worden. Die Obhutspflicht ist nicht so zu verstehen, dass eine Nanny in erster Linie und allein ihren Arbeitgebern verpflichtet wäre; die Obhut ist und bleibt vielmehr dem Kind geschuldet.

Der Vorschlag, das Leihmutterschaftsverhältnis als triadische Beziehung zu verstehen und dementsprechend auszugestalten, kann die Pflicht der Schwangeren, dem Ungeborenen Sorge angedeihen zu lassen, weitaus besser begründen als die Vorstellung der Schwangerschaft als Dienstleistungsverhältnis: Das Kind ist gemäß dem Beziehungsmodell von Leihmutterschaft Teil einer Beziehung, in der sich verschiedene Elternteile in einer auszuhandelnden Weise - in geteilter Elternschaft - um sein Gedeihen bemühen. Während der Schwangerschaft ist es vor allem die Leihmutter, die das Wohl des Fötus befördern kann und muss. Das Beziehungskonzept der Leihmutterschaft kann aber nicht nur die mütterliche Sorgepflicht adäquat begründen; es vermag auch der mütterlichen Bindung zum Fötus, die viele Schwangere empfinden, besser Rechnung zu tragen als Konzepte der Leihmutterschaft als Dienstleistungsverhältnis. Diese emotionale Mutter-Kind-Bindung ist für das Beziehungsmodell von Leihmutterschaft nämlich insofern kein Problem, als sie die angemahnte Beziehung zwischen Tragemutter und Kind natürlicherweise entstehen lässt. Leihmütter, die ihre Tätigkeit als reine Arbeit und sich selber nicht als Teil der triadischen Beziehung verstehen, versuchen oft bereits während der Schwangerschaft, sich gegen eine emotionale Bindung zum Kind zu wehren. So soll ihnen der spätere Abschied vom Baby möglichst leicht fallen. ${ }^{38}$ Die emotionale Distanzierung der Leihmutter von ihrem Baby wird zum Teil von entsprechenden Vermittlungsorganisationen sogar vertraglich gefordert. ${ }^{39}$ Dies ist vor dem Hintergrund des Beziehungsmodells umso tragischer, als die emotionale Bindung zwischen der Schwangeren und dem Ungeborenen zumindest aus Sicht des entstehenden Kindes positiv zu bewerten ist. Entwicklungspsychologischen Studien zufolge gedeihen Babys besser, wenn sie bereits im Mutterleib und kurz nach der Geburt die emotionale Zuwendung - pränatales und perinatales Bonding - ihrer leiblichen (in diesem

37 Vgl. ANDREWS 1995, 13.

38 Vgl. RAgONÉ 1994.

39 Vgl. Hanig 2011, 147. 
Fall austragenden) Mutter erfahren. ${ }^{40}$ Allerdings ließe sich gegen die These der Wichtigkeit der Beziehung der Leihmutter zum Kind argumentieren, dass Adoptivkinder dieses Bonding vermutlich oft auch nicht erlebt haben, weil Frauen, die ihre Kinder zur Adoption freigeben, sich innerlich vermutlich ebenso vom Baby distanzieren wie eine Leihmutter in einem Dienstleistungsverhältnis. Niemand würde jedoch behaupten, alle Adoptivkinder würden deshalb frühkindlich Schaden nehmen. Wie auch immer man den Einfluss des Bondings bewerten will, unterscheiden sich die beiden Fälle Adoption und Leibmutterschaft allerdings in einem wichtigen Punkt: Im Falle einer Adoption wird das ungewollt entstandene Leben geschützt, und es wird versucht, ihm durch die Adoption das bestmögliche Leben zu bieten - als Alternative zum Schwangerschaftsabbruch. Im Fall der Leihmutterschaft hingegen geht es um die Zeugung eines Kindes, das ohne Leihmutter gar nicht entstehen würde. ${ }^{41}$ Die Schwangerschaft lässt sich deshalb in letzterem Fall durchaus so gestalten, dass das Kind optimale Startchancen erhält, etwa indem die Leihmutter frei ist, im Rahmen der geteilten Elternschaft eine Beziehung zum Kind aufzubauen. Das Konzept der Leihmutterschaft als persönliche triadische Beziehung, in der Bonding möglich und erwünscht ist, ist mit Blick auf das Kindeswohl deshalb dem Modell der Leihmutterschaft als Erwerbsarbeit vorzuziehen.

Nehmen wir das Verhältnis zwischen den sozialen Eltern und der Leihmutter in den Blick, so bedingt der Umstand, dass dieses Verhältnis als ein persönliches gestaltet wird, dass die Leihmutter erstens nicht via einer Agentur anonym gebucht wird, sondern dass sich die Beteiligten persönlich kennenlernen und den Kontakt während der Schwangerschaft und auch längerfristig pflegen. Die zukünftigen Eltern betrachten die Leihmutter in einem solchen Verhältnis als eine Person, die sich gemeinsam mit ihnen auf das Projekt einer Schwangerschaft und einer Geburt einlässt, aus dem ein Kind hervorgehen wird, zu dem sie alle eine Beziehung haben. Sie teilen sich die Elternschaft, auch wenn die Rollen, die jedes Elternteil innehaben wird, schon vor der Schwangerschaft abgesprochen und zugewiesen sein können. ${ }^{42}$ Das mag im Zusammenhang mit Elternschaft irritierend klingen, entspricht aber der Realität von geteilter Elternschaft, wie sie zuhauf in Patchwork- und Regenbogenfamilien gelebt wird. In einem solchen Setting der Leihmutterschaft als triadische Beziehung ist die Leihmutter keine zu mietende Gebärmutter, sondern eine Frau, die maßgeblich daran beteiligt ist, dass der Kinderwunsch des werdenden Elternpaars in Erfüllung geht, und die ihre Rolle als eine Form von Mutterschaft ausfüllen kann und will. Die Leihmutter wird deshalb in einer Ersatzmutterschaft als triadische Bezie-

40 Vgl. Kennel, McGrath 2005, 775 und Amendt 1992. Vgl. dazu auch die Richtlinien zur Durchführung der assistierten Reproduktion des WBR der BÄK von 1998. Es ist allerdings unklar, ob die Güter, um die es beim Bonding geht, für die Entwicklung eines Kleinkindes tatsächlich so wichtig sind wie zuweilen suggeriert wird oder ob diese Güter überbewertet werden. Diese Frage kann an dieser Stelle nicht beantwortet werden.

41 So auch TIEU 2009, 172; BER 2000, 161.

42 Für eine Darstellung einer solchen Leihmutterschaft, siehe THERNSTROM 2011. 
hung zweitens als austragende Mutter respektiert, die über ihren schwangeren Körper und das Baby, das in ihr heranwächst, während der Schwangerschaft autonom verfügt. Das bedeutet, dass sie mitentscheiden können muss, welche Untersuchungen sie am Fötus vornehmen lassen will und was im Falle der Feststellung einer Behinderung mit dem Fötus geschehen soll. Könnte das Auftrag gebende Paar die Leihmutter zwingen, eine Schwangerschaft abzubrechen oder bei einer Mehrlingsschwangerschaft ${ }^{43}$ einen der Föten entfernen zu lassen, würde die Leihmutter in der Tat zum Brutkasten degradiert: Sie hat dann keine Stimme hinsichtlich dessen, was mit dem Kind in ihrem Körper geschehen soll, und ihre Selbstverfügungsrechte werden missachtet.

Werden Leihmutterschaftsverhältnisse als persönliche Beziehungen gestaltet, werden sie auch nicht vom Einwand tangiert, dass in ihnen die unveräußerbaren Rechte von Leihmüttern als veräußerbar gelten. Dies behauptet etwa Elizabeth Anderson, die argumentiert: „Pregnancy contracts treat the mother's inalienable right to love her child, and to express that love by asserting a claim to custody in its own best interests, as if it were alienable in a market transaction. "44 Doch Leihmutterschaftsverhältnisse müssen keineswegs die Rechte der austragenden Mutter veräuBern, sondern sie können und sollen so geregelt werden, dass die Leihmutter nach der Geburt nicht gezwungen wird, ihr Kind abzugeben. Zwar wäre es auch für die Bestelleltern vermutlich äußerst schwierig, wenn die Leihmutter ihr Kind behalten möchte. ${ }^{45}$ Doch erstens verlangt der Respekt vor der Würde der Leihmutter, diese Frau nicht allein als Gebärmutter zu sehen, die nach getaner Arbeit die Frucht ihres Körpers abzugeben hat. Vielmehr muss sie auch als eine Mutter gesehen werden, die zum Neugeborenen eine Beziehung aufgebaut hat. Zweitens legt das hier verteidigte Verständnis der triadischen Beziehung nahe, dass eine Übergabe des Kindes, die als trennscharfer Abschied $\mathrm{zu}$ werten ist, nicht stattfindet. Leihmutter und soziale Eltern werden sich idealerweise vielmehr über ein Arrangement verständigen, das es allen erlaubt, die Beziehung zum Kind und zueinander möglichst langfristig auszugestalten. Das Modell der Leihmutterschaft als persönliche triadische Beziehung begegnet damit auch der Gefahr, dass Leihmütter, die im Leben der von ihnen aus-

${ }^{43}$ Viele Leihmütter sind mit Zwillingen schwanger, weil ihnen der Erfolgsrate wegen mehrere Embryonen eingesetzt werden. Wenn das auftraggebende Paar keine Zwillinge wünscht, muss geklärt werden, wer darüber entscheiden darf, wie viele Kinder ausgetragen werden. Für eine Beschreibung eines entsprechenden Falls siehe TIEU 2009, 173.

44 ANDERSON 2000, 23.

45 In der Praxis tritt dieser Fall offenbar selten ein; vgl. dazu ANDREWs 1995, 10, sie schreibt, dass 75\% der Mütter, die ihr Kind zur Adoption frei geben wollen, nach der Geburt anders entscheiden, im Unterschied zu 1\% der Leihmütter. Andere Studien gehen von höheren Zahlen aus. Für weitere Fälle von Sorgerechtsstreitereien um Kinder von Leihmüttern, siehe die Website der AMERICAN SOCIETY OF REPRODUCTIVE MEDICINE (ASRM). 
getragenen Kinder und der intendierten Eltern keine weitere Rolle mehr spielen, unter einem Kontaktabbruch leiden müssen. ${ }^{46}$

Das Kind hat in diesem Konzept der Leihmutterschaft als persönliche triadische Beziehung mehrere Elternteile: Es hat einen Vater und eine Mutter (oder zwei Mütter oder zwei Väter im Falle eines gleichgeschlechtlichen Paares), die sich das Sorgerecht teilen; und es hat eine zusätzliche Mutter - seine Leihmutter. Je nach Typ der Leihmutterschaft (siehe Abschnitt 1) kommen zwei genetische Elternteile hinzu. Dass Kinder mehr als zwei Elternteile haben können, ist nicht erst seit dem Aufkommen von Leihmutterschaft möglich: Bereits die Freigabe zur Adoption, die Samen- und Eizellspende sowie die Patchworkfamilie, in denen Stiefeltern zu den genetischen Elternteilen hinzukommen, haben das Verständnis von Elternschaft und Familie maßgeblich erweitert, und dieser Prozess ist keineswegs abgeschlossen. ${ }^{47}$ Die geteilte Elternschaft wird damit für immer mehr Kinder Realität. Kinder brauchen verlässliche Bezugspersonen - sozial wie rechtlich. So lange sie diese haben, brauchen neue Familienmodelle nicht zu verunsichern. Denn ob verlässliche Bezugspersonen in einer Familie vorhanden sind, entscheidet sich nicht am Umstand, ob sich zwei, drei oder vier Personen die Elternschaft teilen; noch daran, ob die Kinder bei ihren genetischen Eltern aufwachsen. ${ }^{48}$ Entscheidend ist vielmehr, ob die beteiligten Erwachsenen ihren Fürsorgepflichten dem Kind gegenüber während der Schwangerschaft, nach der Geburt und in der Kindheit und Jugend in einem verlässlichen und nachvollziehbaren Arrangement nachkommen. Allerdings sollte dem Kind das Recht zugebilligt sein, seine Bezugspersonen auch dann weiter zu sehen, wenn die Beziehung zwischen den Erwachsenen zerbricht. Als Teil des Beziehungsgefüges hat auch das Kind ein Recht, seine Beziehungen so zu gestalten, wie es sich dies wünscht. Außerdem fordert die Idee der Leihmutterschaft als triadische Beziehung auch Transparenz gegenüber dem Kind hinsichtlich seiner Herkunft - genetisch, wie auch biologisch, also auch hinsichtlich der Identität seiner Leihmutter, denn auch die Leihmutter ist als ein Elternteil zu verstehen. In Anlehnung an Artikel 7 der UN-KRK (Kinderrechtskonvention) wird in den Ländern, welche die Konvention ratifiziert haben, verlangt, dass das Kind bei Volljährigkeit über die genetische Identität seiner Eltern Aufklärung erhalten kann. Im Zuge der Ratifizierung dieses Artikels wurden u. a. in vielen Ländern anonyme Samenspenden verboten. Eine Leihmutterschaft scheint mir für das Leben eines Kindes nicht minder prägend; die Identität der Leihmutter sollte dem betreffenden Kind deshalb ebenso offen gelegt werden. ${ }^{49}$ Das würde auch den Blick dafür schärfen, dass Leihmutterschaften nicht leicht zu nehmende Verhältnisse sind, sondern schwerwiegende Entscheidungen, die das Leben vieler Personen nachhaltig beeinflussen. Das spricht keinesfalls gegen sie, aber für eine besondere Sorgfalt im Umgang mit ihnen.

\footnotetext{
46 Vgl. für eine Zusammenstellung entsprechender Studien VAN DEN AKKER 2007.

47 Vgl. dazu auch die weitreichende Analyse von ARCHARD 2010.

48 Vgl. zu dieser These auch HASLANGER 2009.

49 Dies fordert auch WiesEmann 2006, 147.
} 
Gegen dieses Konzept der Leihmutterschaft als triadische Beziehung lassen sich verschiedene Einwände vorbringen, von denen ich drei abschließend erwähnen möchte:

Erstens kann man die Idee der Leihmutterschaft als persönliche Beziehung für schlichtweg überzogen halten. Denn eine persönliche Beziehung suggeriere, dass sie um ihretwillen, und nicht allein um eines bestimmten Zweckes willen geschätzt werde. Dies unterscheidet sie denn auch von einem Dienstleistungs- oder Auftragsverhältnis. Engagiert etwa ein Paar eine Architektin, die ihr Traumhaus bauen soll, werden die drei Personen zwar lange Zeit intensiv zusammenarbeiten und eine Herzensangelegenheit teilen. Ist das Haus jedoch errichtet, scheint es vollkommen unproblematisch, wenn der Kontakt zwischen den Parteien abbricht; schließlich hat die Beziehung ihren Zweck erfüllt. Tatsächlich meine ich, dass sich Leihmütter von Architektinnen und anderen Arbeitnehmenden in moralisch bedeutsamer Weise unterscheiden: Einerseits weil Leihmütter, wie ich oben bereits ausgeführt habe, ihren Körper in besonderer Art und Weise in die Arbeit einbringen und deshalb Gefahr laufen, in ihrer Würde verletzt zu werden, wenn sie auf ihre Körperfunktion reduziert werden und als nicht mebr gebraucht entlassen sind, sobald das Kind geboren ist. Andrerseits hat auch die Leihmutter zum werdenden Kind ein anderes Verhältnis als die Architektin zum Haus: Sie lebt mit dem Kind in einer Verbindung, wie sie intimer nicht sein könnte. Ein Leihmutterschaftsverhältnis als persönliche triadische Beziehungen zu verstehen, verlangt von allen Beteiligten, sich in einer ganz anderen Weise auf dieses Verhältnis einzulassen als dies bei einem reinen Vertragsverhältnis der Fall ist. Damit ist zwar noch nichts darüber ausgesagt, wie diese Beziehung vor der Geburt ausgestaltet und nach der Geburt gelebt werden soll; das wird sich im Laufe der Beziehungsgeschichte weisen. Ich habe an dieser Stelle nicht behauptet, dass die Involvierten den Kontakt pflegen sollten bis ans Ende ihres Lebens. Keine Beziehung trägt in sich eingeschrieben ihren Gang; der Lauf ihrer Geschichte und deren Ende sind immer offen. Jedoch sind die Suche nach einer Leihmutter, die Einpflanzung des Embryos sowie Schwangerschaft und Geburt des Kindes anders anzugehen, wenn die Leihmutterschaft als Teil einer Beziehungsgeschichte verstanden wird, als wenn sie im Rahmen eines Arbeitsverhältnisses zustande kommen.

Zweitens kann gegen das hier vorgeschlagene Modell eingewendet werden, gerade in persönlichen Beziehungen sei die Gefahr von Abhängigkeit und Ausbeutung besonders groß. Das hier nahe gelegte Modell berge deshalb mehr Risiken als Leihmutterschaften, die als Dienstleistungsverhältnisse konzipiert würden. Auch wenn ich zustimme, dass es naiv wäre zu meinen, das Bestehen einer persönlichen Beziehung diene als Bollwerk gegen Ausbeutung, glaube ich nicht, dass damit das vorgeschlagene Modell in Frage gestellt wird. Denn vorgeschlagen wurde ja nicht, dass sich kinderlose Paare mit ihrem Wunsch nach Nachwuchs in bestehenden Beziehungen umsehen und sich etwa an Freundinnen oder Schwestern wenden, die möglicherweise Mühe bekunden, dem Wunsch eine Absage zu erteilen. Eine solche Forderung könnte in der Tat erhebliches Missbrauchspotential bergen. Das Modell schlägt vielmehr vor, dass sich intendierte Eltern und Frauen, die sich vorstellen könnten, Leihmutter zu werden, kennenlernen und sich aufeinander einlassen und 
im Laufe ihrer wachsenden persönlichen Beziehung entscheiden, ob sie bereit sind, sich dem Wagnis eines gemeinsamen Kindes zu stellen.

Ein dritter Einwand lautet, Leihmutterschaftsverhältnisse, wie hier skizziert, würden die Beteiligten emotional überfordern. Schließlich handelt es sich notgedrungen um eine Dreiecksbeziehung, in der es zu Eifersucht und Sorgerechtsstreiten kommen kann. Diesen Punkt der emotionalen Ambitioniertheit gestehe ich unumwunden zu. Die Frage ist allerdings, was die Alternativen zu meinem zugegeben anspruchsvollen Modell sind: Wir können entweder Leihmutterschaften dulden und fördern, in denen viele Gefahren lauern, dass die betreffenden Frauen ausgebeutet oder instrumentalisiert werden und dass die entstehenden Kinder ihre Leihmütter nie kennenlernen werden; oder - wenn wir die Alternative für überfordernd halten auf diese Praxis ganz verzichten. Ich plädiere hier für einen dritten Weg: Sich bewusst zu machen, dass Leihmutter zu werden und eine Leihmutter zu suchen moralisch äußerst sensible Verfahren sind, in denen Menschen sich auf eine komplexe und schwierige Beziehung einlassen, die des besonderen rechtlichen Schutzes und der speziellen gesellschaftlichen Begleitung bedarf. Unser Ziel muss es - mit Claudia Wiesemann gesprochen - sein, eine „Strategie der Vollständigkeit“50 zu verfolgen: die geteilte Elternschaft zu fördern, in der alle ihre Rolle haben und gemeinsam das Kindswohl im Blick haben, selbst über Leihmutterschaftsverhältnisse hinweg. Um das Eingangszitat aufzugreifen: Kinderkriegen mag im globalen Zeitalter und vor allem im Zeitalter der modernen Reproduktionsmedizin auch zu einer Frage der Logistik werden - vor allem muss sie aber immer eine Frage der Beziehungen bleiben, in die neues Leben hineingeboren wird.

\section{Literatur}

AMENDT, G. (1992): Das Leben unerwünschter Kinder, Frankfurt a.M.

Anderson, E. (1990): Is Women's Labor a Commodity, in: Philosophy and Public Affairs 19 (1), 71-92.

ANDERSON, E. (2000): Why Commercial Surrogate Motherhood Unethically Commodifies Women and Children: Reply to McLachlan and Swales, in: Health Care Analysis 8, 19_ 26.

50 WIESEMANN 2006, 145.

* Für hilfreiche Kommentare danke ich Norbert Anwander, Monika Betzler, Anca Gheaus, Magdalena Hoffmann, Jörg Löschke, Peter Schaber, Jean-Daniel Strub und zwei anonymen Gutachtern. Für die finanzielle Unterstützung der Arbeit an diesem Beitrag danke ich dem Schweizerischen Nationalfonds SNF. 
ANDREWs, L.B. (1995): Beyond doctrinal boundaries. A legal framework for surrogate motherhood, in: Virginia Law Review 81, 2343-2375.

ArCHARD, D. (2010): The family. A liberal defence, Basingstoke.

BER, R. (2000): Ethical Issues in Gestational Surrogacy, in: Theoretical Medicine and Bioethics 21, 153-169.

Buchanan, A. (1985): Ethics, Efficiency, and the Market, Totowa N.J.

CAHILL, L.S. (1990): The ethics of surrogate motherhood. Biology, Freedom, and Moral Obligation, in: GosTiN L. (ed.): Surrogate motherhood. Politics and privacy, Bloomington, Indianapolis, 151-164.

CAPREZ, C. (2012): Familienbande, Zürich.

ERLINGER, R. (2011): Ist Leihmutterschaft Sünde?, in: Geo 12, 156.

Field, M. (1989): Surrogate Motherhood, Cambridge.

GHEaus, A. (2011): The Right to Parent One's Biological Baby, in: Journal of Political Philosophy vom 27. April 2011.

HANIG, F. (2011): Eine andere Frau trägt unser Kind aus, in: GEO 12, 140-162.

Haslanger, S. (2009): Family, Ancestry and Self. What is the Moral Significance of Biological Ties?, in: Adoption \& Culture 2, 91-122.

HiLl, T. (1992): Humanity as an End in Itself, in: HiLl, T. (eds.): Dignity and Practical Reason, Ithaca, 38-57.

Honneth, A., Rössler, B. (eds.) (2008): Von Person zu Person. Zur Moralität persönlicher Beziebungen, Frankfurt a.M.

HÖRnLE, T. (im Druck): Menschenwürde und Ersatzmutterschaft, in: JOERDEN, J.C., Hilgendorf, E., Thiele, F. (Hrsg.): Menschenwürde und Medizintechnik, Berlin.

KANT, I. (1785): Grundlegung zur Metaphysik der Sitten, Akademie-Ausgabe, Bd. IV, Berlin.

- (1797): Metaphysike der Sitten, Akademie-Ausgabe, Bd. VI, Berlin.

Keller, R., GÜNTHER, H.L., KAISER, P. (1992): Embryonenschutəgesetz: Kommentar zum Embryonenschutzgesetz, Stuttgart.

KENNELL, J., MCGRATH, S. (2005): Starting the process of mother-infant bonding, in: Acta Paediatrica 94, 775-778.

KersteIN, S.J. (2009): Kantian Condemnation of Commerce in Organs, in: Kennedy Institute of Ethics Journal 19 (2), 147-169.

MCLACHLAN, H.V. (1997): Defending commercial surrogate motherhood against Van Niekerk and $V$ an $Z y l$, in: Journal of Medical Ethics 23 (6), 344-348. 
Muldin, A. (2005): Reconceiving Pregnancy and Childcare. Ethics, Experience, and Reproductive Labor, Cambridge.

Pateman, C. (1983): Defending Prostitution. Charges against Ericson, in: Ethics 93, 561565.

PuRDY, L.M. (1992): Another look at contract pregnancy, in: HoLMES, H.B. (ed.): Issues in Reproductive Technology. An Anthology, New York, London, 309-311.

Ragoné, H. (1994): Surrogate motherhood. Conception in the Heart, Boulder, San Francisco, Oxford, 75-78.

SCHABER, P. (2010): Instrumentalisierung und Würde, Paderborn.

- (2012): Menschenwürde, Stuttgart.

Shrage, L. (2007): Feminist Perspectives on Sex Markets, in: Stanford Encyclopedia of Philosophy, URL http://plato.stanford.edu/entries/feminist-sex-markets/ (Stand: 23.07.2012).

TEMAN, E. (2010): Birthing a mother. The surrogate body and the pregnant self, Berkeley.

Thernstrom, M. (2011): Vater, Mutter, Mutter, Mutter, Mutter, Kind, Kind, in: Nido 0 vom 1.4.2011, 64-71.

Thomson, J.J. (1971): A Defence of Abortion, in: Philosophy \& Public Affairs 1, 4766.

TIEU, M.M. (2009): Altruistic surrogacy. The necessary objectification of surrogate mothers, in: Journal of Medical Ethics 35, 171-175.

TONG, R. (2003): Surrogate Motherhood, in: Frey, R.G., Wellman, C.H. (eds.): A Companion to Applied Ethics, Oxford, 369-381.

Tormey, J. F. (1974): Exploitation, Oppression and Self-Sacrifice, in: Philosophical Forum 5, 206-221.

VAN DEN AKKER, O. (2003): Genetic and gestational surrogate mothers' experience of surrogacy, in: Journal of Reproductive and Infant Psychology 21 (2), 145-161.

- (2005): A longitudinal pre pregnancy to post delivery comparison of genetic and gestational surrogate and intended mothers. Confidence gynecology, in: Journal of Psychosomatic Obstetrics and Gynecology 26 (4), 277-284.

- (2007): Psychosocial aspects of surrogate motherhood, in: Human Reproduction Update 13 (1), 53-62.

VAN NIEKERK, A. / VAN ZYL, L. (1995): The ethics of surrogacy. Women's reproductive labour, in: Journal of Medical Ethics 21 (6), 345-349.

- (2000): Interpretations, perspectives and intentions in surrogate motherhood, in: Journal of Medical Ethics 26 (5), 404-409. 
WertheImer, A. (2008): Exploitation, in: Zalta, E.N. (ed.): The Stanford Encyclopedia of Philosophy, http://plato.stanford.edu/entries/exploitation/ (Stand: 23.07.2012).

WiESEMANN, C. (2006): Von der Verantwortung, ein Kind zu bekommen. Eine Ethik der Elternschaft, München.

WiLLmOTT, L. (2002): Surrogacy. Ill-conceived rights, in: Journal of Law and Medicine 10 (2), 198-220.

WISSENSCHAFTLICHER BEIRAT (WBR) der Bundesärztekammer (BÄK) (1998): Richtlinien zur Durchführung der assistierten Reproduktion, in: Deutsches Ärzteblatt 95 (49), A-3166-A-3171. 
